\title{
Study on Rotating Compression Forming of Double- layer Clad Ring Considering Coulomb Friction
}

\author{
Gow-Yi Tzou',, Yeong-Maw Hwang', and Hsiang-Yu Teng' \\ 1 Department of Mechanical and Automation Engineering, Kao Yuan University, Kaohsiung City, 821, Taiwan, R.O.C \\ 2 Department of Mechanical and Electro-Mechanical Engineering, National Sun Yat-Sen University, Kaohsiung City, 804, Taiwan, R.0.C \\ \# Corresponding Author / E-mail: gowyitzou@cc.kyu.edu.tw, TEL: +886-7-6077990, FAX: +886-7-6976206
}

KEYWORDS: Coulomb friction, Rotating compression forming, Double-layer bounded clad ring, FEM simulation

This study uses two FEM simulations based on deform $3 D$ and superform commercial software assuming coulomb friction to explore the plastic behavior in rotating compression forming of double-layer bounded clad ring. The rotating compression forming can reduce the compressive force and make the deformation uniform. Effects of the frictional coefficient, shear yield stress ratio, rotating angular velocity, frictional coefficient ratio, clad ring height ratio etc on compression characteristics including the effective stress, the effective strain, the velocity field, the shear strain, the compressive force etc. are investigated effectively. Moreover, the realistic rotating compression experiments of clad ring have been carried out to verify the acceptance of both FEM simulation models.

Manuscript received: April 10, 2014 / Revised: May 12, 2014 / Accepted: May 22, 2014

\section{NOMENCLATURE}

$$
\begin{aligned}
& \mu=\text { Coulomb frictional coefficient } \\
& \mathrm{r}=\text { reduction ratio }(\%) \\
& \omega=\text { rotating angular velocity }(\mathrm{rad} / \mathrm{sec}) \\
& \mathrm{V}=\text { top die velocity }(\mathrm{mm} / \mathrm{sec}) \\
& \mathrm{D}_{\mathrm{o}}=\text { initial outer diameter of clad ring }(\mathrm{mm}) \\
& \mathrm{D}_{\mathrm{i}}=\text { initial inner diameter of clad ring }(\mathrm{mm}) \\
& \mathrm{H}_{\mathrm{i}}=\text { whole height of double-layer clad ring }(\mathrm{mm}) \\
& \mathrm{E}=\text { Young's modulus } \\
& \nu=\text { Poisson's ratio } \\
& \sigma=\text { flow stress }
\end{aligned}
$$

\section{Introduction}

With a view to solving the problems occurred in cylinder and ring compression forming, for example the non-uniform deformation and crack in the internal and external parts of materials; the bulging of outer and inner surfaces. Many researchers have been doing the related studies to analyze the compressive force, material flow, stress and strain distributions etc. in compression forming. Douglas et al. ${ }^{1}$ uses the ring compression test to predict the flow stresses under various forging rates and temperatures. Sofuoglu et al. ${ }^{2}$ utilized the ring compression test to obtain the friction calibrating curve based on the ABAQUS FEM simulation of elasto-platic material in order to measure frictional coefficient. Xua et al. ${ }^{3}$ used rigid-plastic FEM to analyze the rotating compression of cylinder, it indicates the compression force can be reduced and especially for the thinner workpiece the non-uniform deformation can be decreased using the rotating compression. Kim et al. ${ }^{4}$ established 3D kinematic admissible velocity field of rotating cylinder compression based on the upper bound method considering constant shear friction to explore the plastic behaviors; it is noted the forging force and bulging can be reduced by the rotating forging process. Kim et al. ${ }^{5}$ used the dual-stream function method to construct the 3D kinematic admissible velocity field to investigate the plastic behavior in rotating cylinder compression; this analysis model is more accurate than the upper bound method. Furthermore Kim et al. ${ }^{6}$ also used the dual-stream function method to explore the plastic behavior with rotating tool and non-rotating workpiece. In 2002 and 2004, Tzou and Huang et al. ${ }^{7-9}$ established three analysis models considering constant shear friction, Coulomb friction, and hybrid friction to explore the various stress distributions and compressive force in rotating cylinder compression; however the yielding criterion didn't consider the hoop frictional shear stress. In 2006, Tzou et al. ${ }^{10}$ performed the analysis and experiment verification of rotating cylinder compression based on the slab method and upper bound method considering 
constant shear friction. In 2007, Tzou et al. ${ }^{11}$ established the slab analysis of rotating cylinder compression with Coulomb friction; the yielding criterion considering hoop frictional shear stress is used to derive the analytical models with rotating and non-rotating effects. Moreover, the compression pressure, the radial stress, the compressive force, the rotating torque can be predicted effectively; it is more accurate than one without considering the hoop frictional stress. In 2007 , the hybrid friction analytical model based on the slab method has been proposed by Tzou et al., ${ }^{12}$ the interface between the dies and cylinder is assumed to be hybrid friction which Coulomb friction and sticking friction are occurred in the contact interface meanwhile. In 2007, Chang and Tzou et al. ${ }^{13}$ proposed the rotating cylinder compression analysis based on the slab method, upper bound method, FEM simulation; besides the realistic experiment has been carried out to verify the acceptance of three analysis models. In 2003, Hsu and Tzou et al. ${ }^{14}$ analyzed the compression characteristics of double-layer clad sheet using the slab method and upper bound method, and compared the variation of both models. In 2004, Alexandrov and Tzou et al. ${ }^{15}$ firstly proposed the rotating compression of ring using the upper bound method, the trend is similar with the rotating cylinder compression. Chen and Tzou et al. ${ }^{16}$ proposed an analytical model based on upper bound method in twist compression of cylinder, and compared to the slab analysis taking into account the hoop frictional shear stress. Solhjoo ${ }^{17}$ used upper bound theory and Deform 2D to evaluate the constant shear frictional factor in the cylinder compression without rotation. Alexandrov ${ }^{18}$ used the upper bound method to explore the plastic behavior of viscous cylinder compression without rotation. Wu et al. ${ }^{19}$ studied effect of natural boundary conditions on the upperbound analysis of upset forging of ring And disks without rotation. Park et al. ${ }^{20}$ proposed a new method of constructing a velocity field is proposed to solve metal forming problems by the upper-bound elemental technique (UBET).

In the present study, the FEM simulations based on Deform 3D and Superform commercial software have been used to explored the plastic behaviors of rotating compression of double-layer clad ring, besides carries out the realistic experiments to verify the acceptances of FEM simulations.

\section{Finite Element Modeling}

In this paper, the commercial software, Deform and Superform, are used to simulate the rotating compression of double-layer clad ring. This software can provide the elastic-plastic and rigid-plastic simulation of the metal forming in large deformation. The FEM software (Deform 3D and Superform) enable to do the tool design and product design. The FEM simulations provide 3D analysis with the tetrahedron (Deform 3D) and hexagonal elements(Superform) for meshing the work-piece of bulk forming and the auto re-mesh step is enveloped in the software package. Using the FEM software, it can significantly reduce the cost and time consumption of tool and die design. The flow pattern, effective stress distribution, effective strain distribution, velocity field of work-piece and load stoke can be simulated by FEM. These simulation results can be used to obtain the product geometric profile and material properties required. In the pre- process of modeling, the geometric profile of double-layer clad ring can be constructed. The rigid-plastic analysis (Deform 3D) and elstoplastic analysis (Superform) in rotating piercing process is chosen and the material properties such as Young's modulus, Poisson's ratio, are needed. For the first stage of exploring the rotating compression of double-layer clad ring, the isotropic work-hardening rule is assumed in flow rule due to plastic strain hardening. The von Mises yield criterion is used. The initial conditions of work-piece are set up, and the contact along work-piece and dies are defined. The punch with a downward speed is $1 \mathrm{~mm} / \mathrm{sec}$, all rigid contact bodies are assumed as insulation, and the heat conduction phenomenon during the double-layer clad ring is neglected. In the rotating compression of double-layer clad ring, the relationship between strain and stress is nonlinear. The DEFORM has been developed to resolve the nonlinear problem. The conventional von Mises yield surfaces is available in DEFORM. The contact bodies are regarded as rigid surface. The Deform preprocess is shown in Fig. 1; the Superform preprocess is shown in Fig. 2.

With a view to simulating the rotating compression of double-layer clad ring, the forming conditions, the geometry conditions, and material conditions are shown in Table 1.

Fig. 3 shows variations of compressive force with reduction ratio for various angular rotating velocities. As the reduction ratio increases, it indicates the working hardening is increased gradually so that the

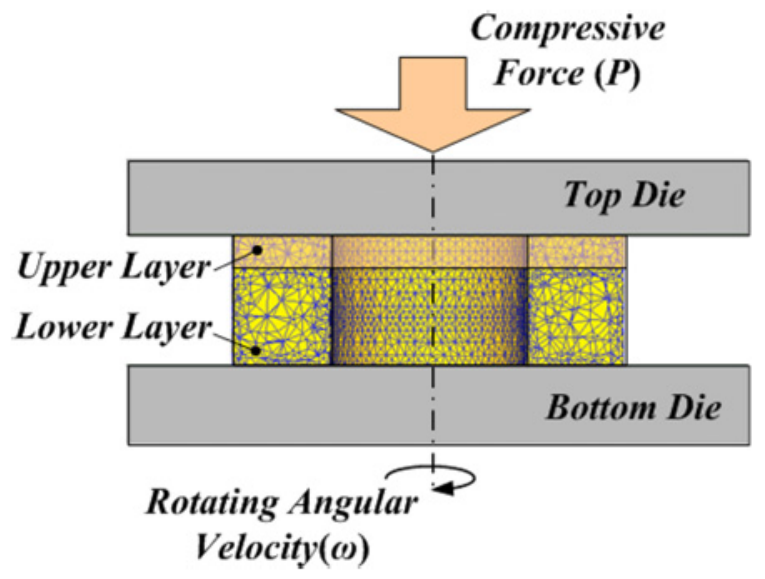

Fig. 1 Schematic diagram of Deform-3D preprocess in rotating compression forming of the double-layer bounded clad ring

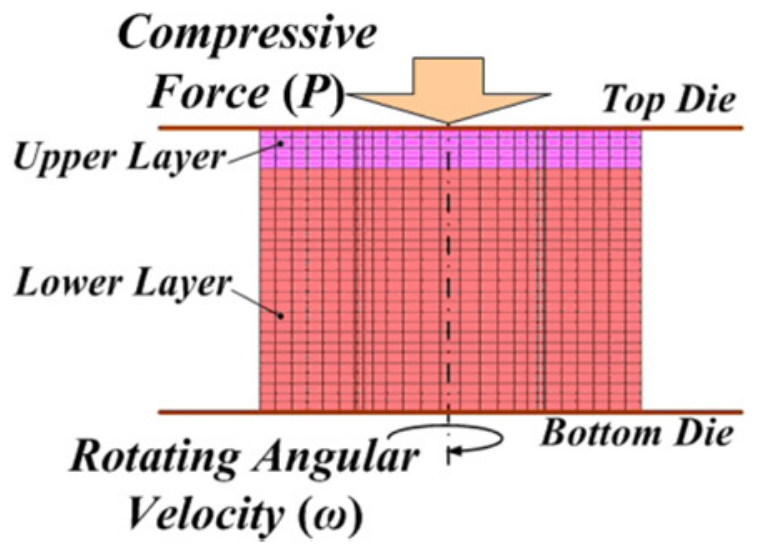

Fig. 2 Schematic diagram of Superform preprocess in rotating compression forming of the double-layer bounded clad ring 
compressive force increases. Applying the rotating angular velocity on the lower die, the material of clad ring is easy to flow so that the compressive force is reduced. At the reduction ratio $38.5 \%$, the compressive force with the rotating effect is lower, $9.56 \%$, than that without rotation. Therefore when forming with rotation; the energy in

Table 1 Simulation conditions of clad ring in rotating compression

\begin{tabular}{|c|c|c|c|}
\hline $\mathbf{D}_{0}$ & & \multicolumn{2}{|c|}{ Forming Conditions } \\
\hline$\stackrel{D_{i}}{\leftrightarrow}$ & \multirow{4}{*}{$=\underbrace{4}_{=}$} & Coulomb friction $(\mu)$ & $0.01-0.3$ \\
\hline \multirow{7}{*}{ SA285 } & & Reduction (r \%) & $10-40$ \\
\hline & & $\begin{array}{l}\text { Rotating Angular } \\
\text { Velocity }(\omega \mathrm{rad} / \mathrm{sec})\end{array}$ & 0,1 \\
\hline & & $\begin{array}{l}\text { Top Die Velocity } \\
\text { (V mm/sec) }\end{array}$ & 1 \\
\hline & & \multicolumn{2}{|c|}{$\begin{array}{c}\text { Geometry Conditions } \\
\end{array}$} \\
\hline & & Outer Diameter $\left(D_{0}\right)$ & $12 \mathrm{~mm}$ \\
\hline & & Inner Diameter $\left(\mathrm{D}_{\mathrm{i}}\right)$ & $6 \mathrm{~mm}$ \\
\hline & & Height $\left(\mathrm{H}_{\mathrm{i}}\right)$ & $14 \mathrm{~mm}$ \\
\hline \multicolumn{4}{|c|}{ Material Conditions } \\
\hline $\begin{array}{c}\text { Material } \\
\text { (Upper layer) }\end{array}$ & SUS304 & $\begin{array}{c}\text { Material } \\
\text { (Lower layer) }\end{array}$ & SA285 \\
\hline $\begin{array}{l}\text { Young's Modulus } \\
\text { (E) }\end{array}$ & $210 \mathrm{GPa}$ & $\begin{array}{l}\text { Young's Modulus } \\
(\mathrm{E}) \\
\end{array}$ & $210 \mathrm{GPa}$ \\
\hline Poisson's Ratio (v) & 0.3 & Poisson's Ratio (v) & 0.3 \\
\hline Flow Stress $(\sigma)$ & $1074.555 \varepsilon^{0.1934}$ & Flow Stress $(\sigma)$ & $840.946 \varepsilon^{0.24}$ \\
\hline
\end{tabular}

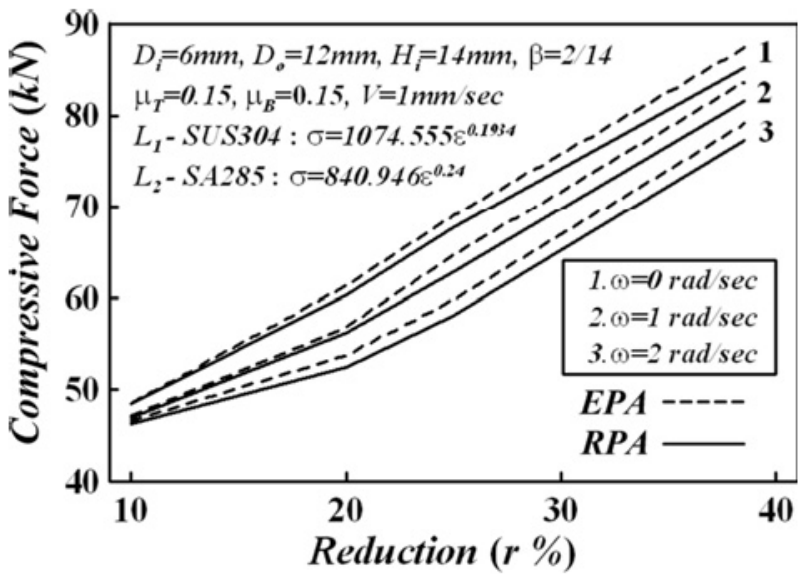

Fig. 3 Variations of compressive force with reduction ratio for various angular rotating velocities

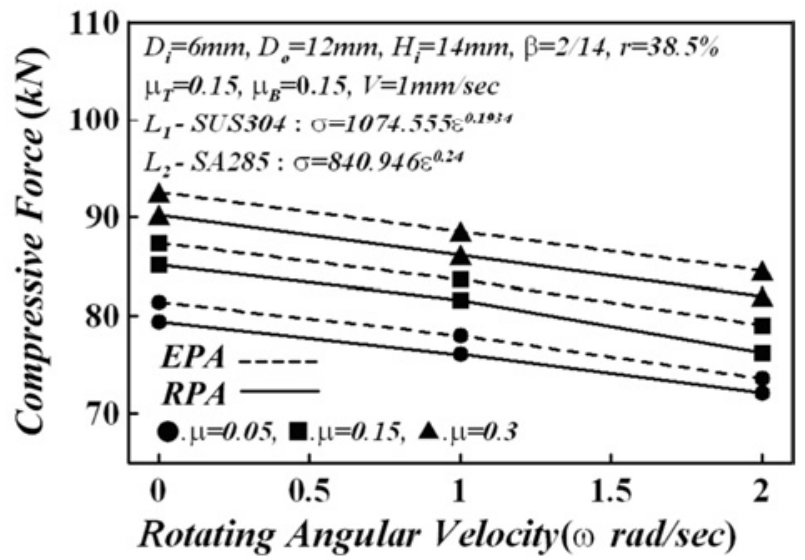

Fig. 4 Variations of compressive force with rotating angular velocity for various frictional coefficients the forming can be saved. From this figure, the compressive force obtained from Superform is slightly higher than that from Deform-3D.

Fig. 4 shows variations of compressive force with rotating angular velocity for various frictional coefficients. It is noted that the compressive force is decreased as the angular velocity increases to raise the torque on the hoop direction. Under the fixed angular velocity, the compressive force is increased as the frictional coefficient increases.

With a view to verifying acceptance and variations of both FEM simulations, the realistic experiment is carried out to confirm the simulation results. The specimen is clad ring which has initial outer diameter $12 \mathrm{~mm}$, initial inner diameter $6 \mathrm{~mm}$, initial height $14 \mathrm{~mm}$, the upper layer is SUS 304, the lower layer is SA285, the clad height ratio $2 / 14$. Some results in the compression forming considering nonrotation and rotation effect are shown in the followings.

The Effects of reduction ratio on compressive force between FEM simulations and experiment in non-rotating compression forming are demonstrated in Fig. 5. From this figure, as the reduction ratio increases, the compressive forces obtained from both FEM simulations and experiment always increase reasonably, and in agreement with the experiment. The error between Deform 3D (Rigid-Plastic Analysis, RPA) and experiment is around 3.86\%; the error between Superform (Elasto-Plastic Analysis, EPA) and experiment is around 3.55\%. The errors are more reasonable in the accepted range, and the acceptances of both simulations can be verified.

With a view to looking at the profiles of outer surface in then onrotating forming with different reduction ratios, results of the experiments and both simulations are shown in Fig. 6. The frictional coefficient of upper layer (SUS 304) is assumed to be 0.2 ; the frictional coefficient is assumed to be 0.1 . The bulging profiles obtained from rigid-plastic analysis and elasto-plastic analysis are similar with the experiments.

Fig. 7 is the outer and inner deformation profiles in the non-rotating forming with different reduction ratios. From this table, because the upper layer (SUS 304) is harder than the lower layer (SA258), the hard layer is inset to the soft layer. The soft layer is easy to be deformed, however the hard layer is difficult to deform so that it insets to the soft layer, as can be seen in experiments and both simulations. Moreover the lower layer has low frictional coefficient, thus the outer and inner surfaces are deformed away from the center of the clad ring. Both simulations are in good agreement with the experiments.

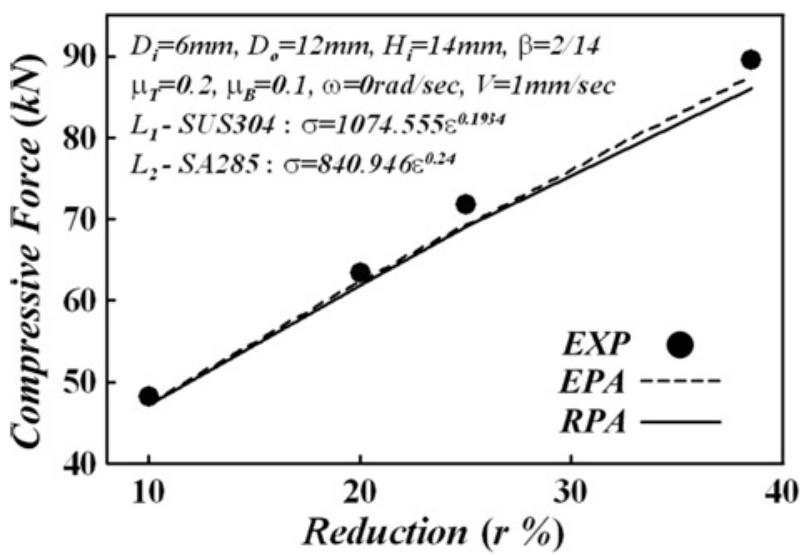

Fig. 5 Effects of reduction ratio on compressive force between FEM simulations and experiment in non-rotating compression forming 
Effective stress and effective strain in the non-rotating forming with different reduction ratios are shown in Fig. 8. At $r=10 \%$, the maximum effective stress obtained from rigid-plastic analysis is $660 \mathrm{MPa}$, that obtained from elasto-plastic analysis is $680.4 \mathrm{MPa}$; the error between both simulations is around $3 \%$. At $r=20 \%$, the maximum effective stress obtained from Rigid-Plastic Analysis is
$870 \mathrm{MPa}$, that obtained from elasto-plastic analysis is $886.4 \mathrm{MPa}$; the error between both simulations is around $1.85 \%$. At $r=25 \%$, the maximum effective stress obtained from rigid-plastic analysis is $1050 \mathrm{MPa}$, that obtained from elasto-plastic analysis is $1059 \mathrm{MPa}$; the error between both simulations is around $0.85 \%$. At $r=38.5 \%$, the maximum effective stress obtained from elasto-plastic analysis is

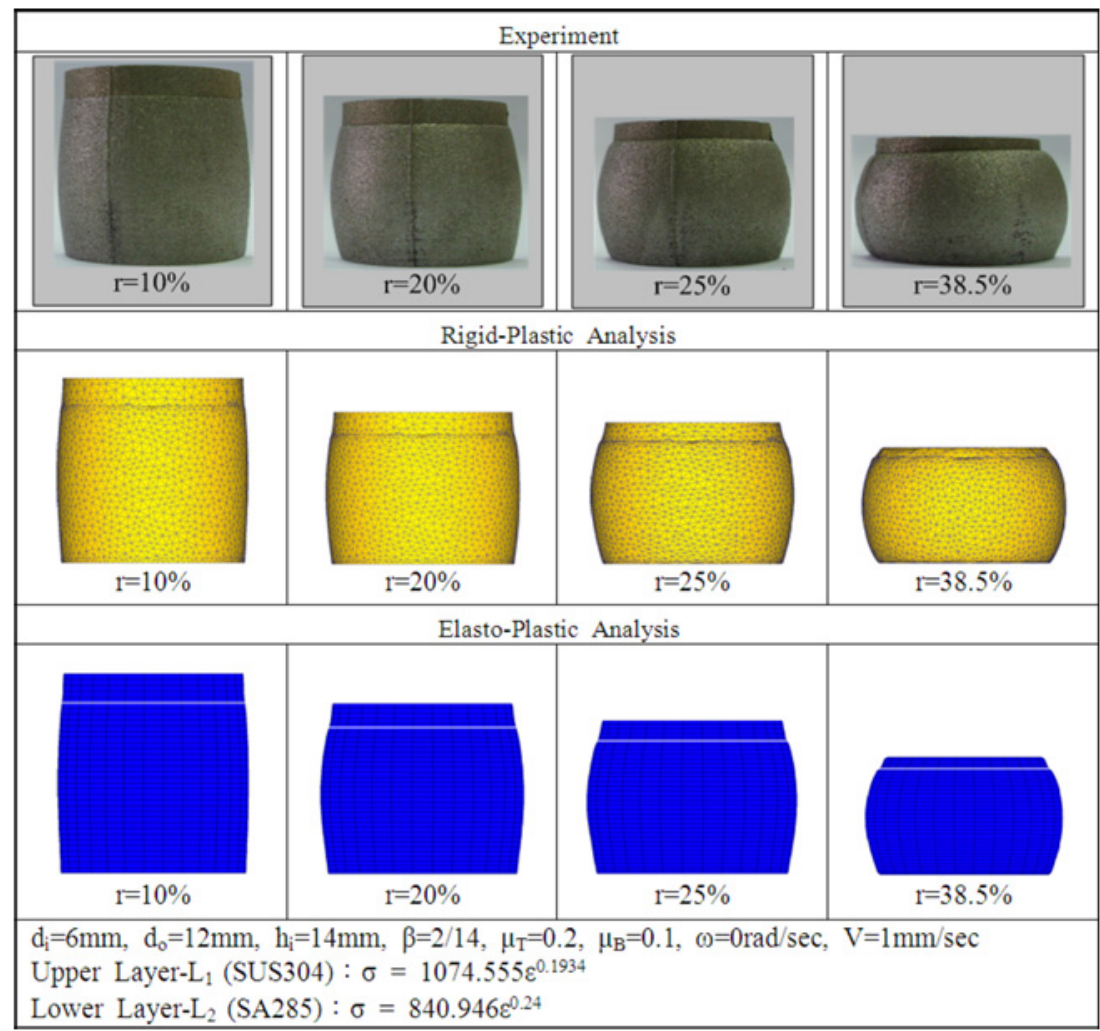

Fig. 6 Bulging profiles of outer surface in the non-rotating forming with different reduction ratios

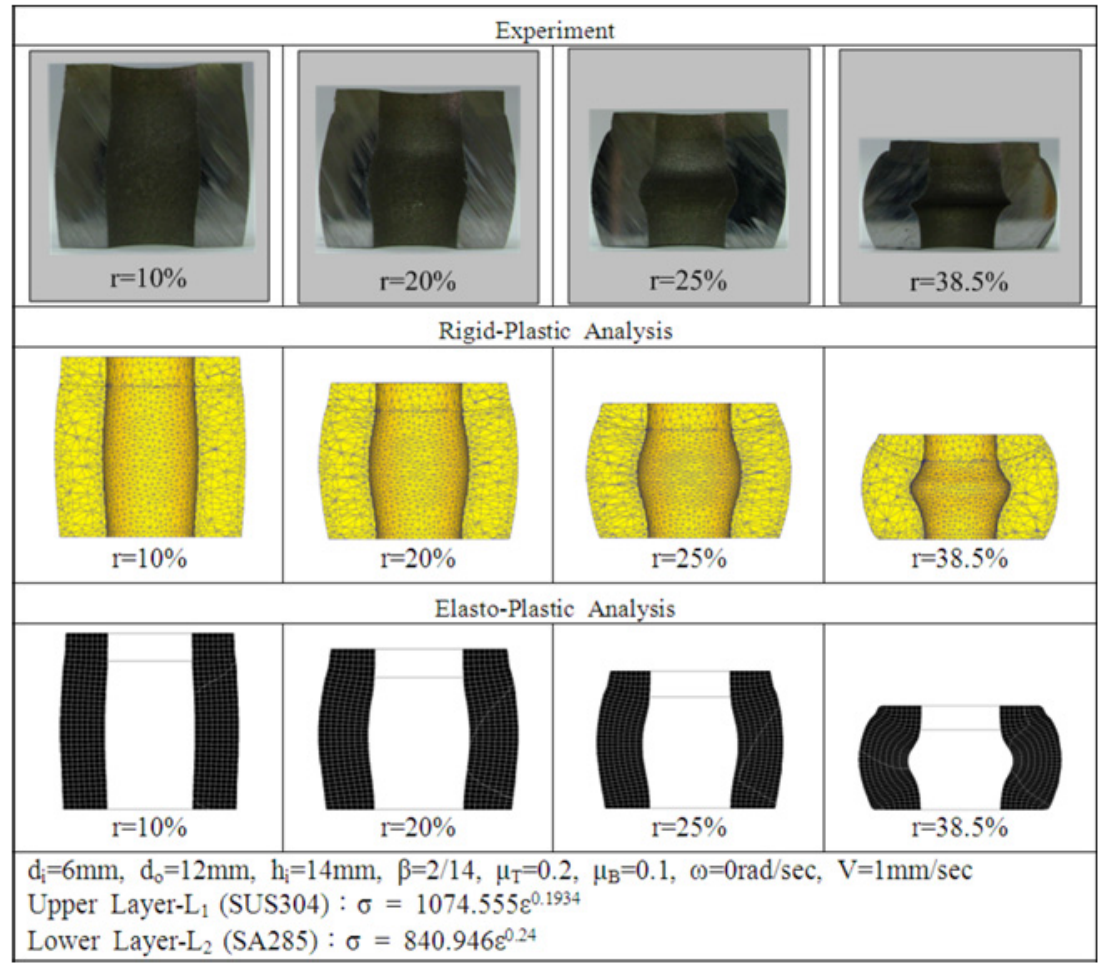

Fig. 7 Outer and inner deformation profiles in the non-rotating forming with different reduction ratios 
$1340 \mathrm{MPa}$, that obtained from elasto-plastic analysis is $1364 \mathrm{MPa}$; the error between both simulations is around $1.75 \%$. Generally the maximum effective stress increases with increasing the reduction ratios. At $\mathrm{r}=10 \%$, the maximum effective strain obtained from rigidplastic analysis is $0.16 \mathrm{~mm} / \mathrm{mm}$, that obtained from elasto-plastic analysis is $0.17 \mathrm{~mm} / \mathrm{mm}$; the error between both simulations is very small. At $\mathrm{r}=20 \%$, the maximum effective strain obtained from RigidPlastic Analysis is $0.51 \mathrm{~mm} / \mathrm{mm}$, that obtained from elasto-plastic analysis is $0.42 \mathrm{~mm} / \mathrm{mm}$. At $\mathrm{r}=25 \%$, the maximum effective strain obtained from elasto-plastic analysis is $0.78 \mathrm{~mm} / \mathrm{mm}$, that obtained from elasto-plastic analysis is $0.6 \mathrm{~mm} / \mathrm{mm}$; the error between both simulations is around $0.85 \%$. At $r=38.5 \%$, the maximum effective strain obtained from rigid-plastic analysis is $1.3 \mathrm{~mm} / \mathrm{mm}$, that obtained from elasto-plastic analysis is $1.33 \mathrm{~mm} / \mathrm{mm}$; the error between both simulations is around $2.5 \%$. The location of maximum effective strain is mainly on the inner surface deformed from the center of clad ring.

Effects of reduction ratio on compressive force between FEM simulations and experiment in rotating compressive forming are demonstrated in Fig. 9. From this figure, as the reduction ratio increases, the compressive forces obtained from both FEM simulations and experiment always increase reasonably, and in good agreement with the experiment. The error between rigid-plastic analysis and experiment is around $5.07 \%$; the error between elasto-plastic analysis and experiment is around $4.75 \%$. The errors are more reasonable in the accepted range, and the acceptances of both simulations can be verified.

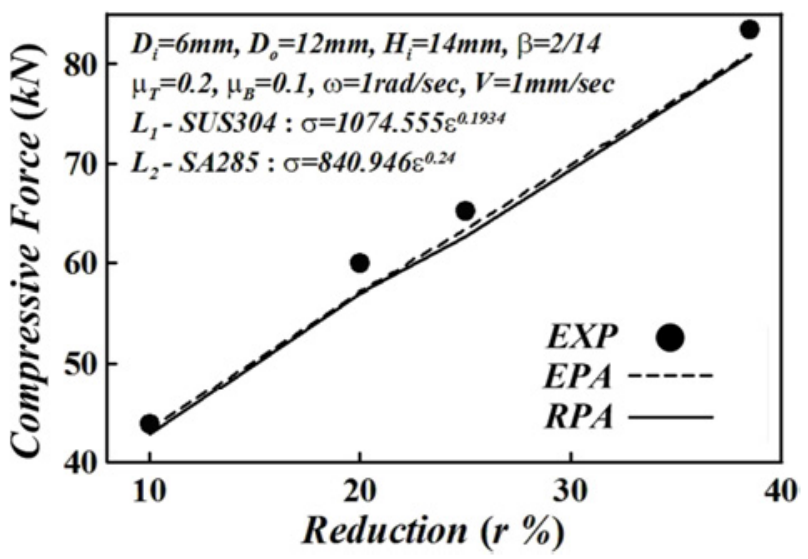

Fig. 9 Effects of reduction ratio on compressive force between FEM simulations and experiment in rotating compression forming

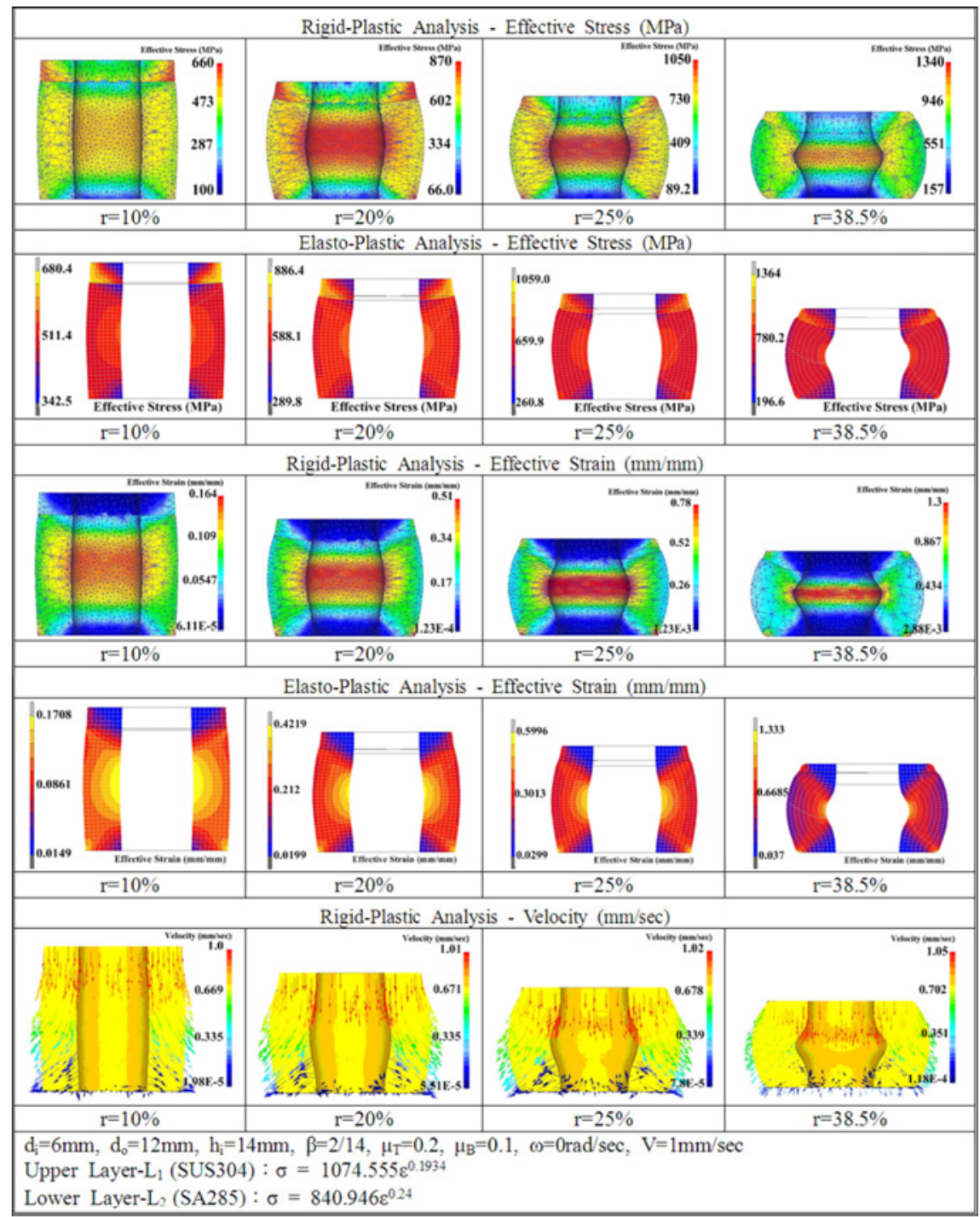

Fig. 8 Effective stress, effective strain in the non-rotating forming with different reduction ratios 
With a view to looking at the profiles of outer surface and shear strain in the rotating forming with different reduction ratios, results of the experiments and both simulations are shown in Fig. 10. The frictional coefficient of upper layer (SUS 304) is assumed to be 0.2 ; the frictional coefficient is assumed to be 0.1 . The bulging profiles obtained from rigid-plastic analysis and elasto-plastic analysis are similar with the experiments. In the rotating forming, due to the lower die's rotation, the shear strain for each layer can be measured from the experiment and elasto-plastic analysis simulation. However, the shear strain is not easy to be measured in rigid-plastic analysis simulation. As

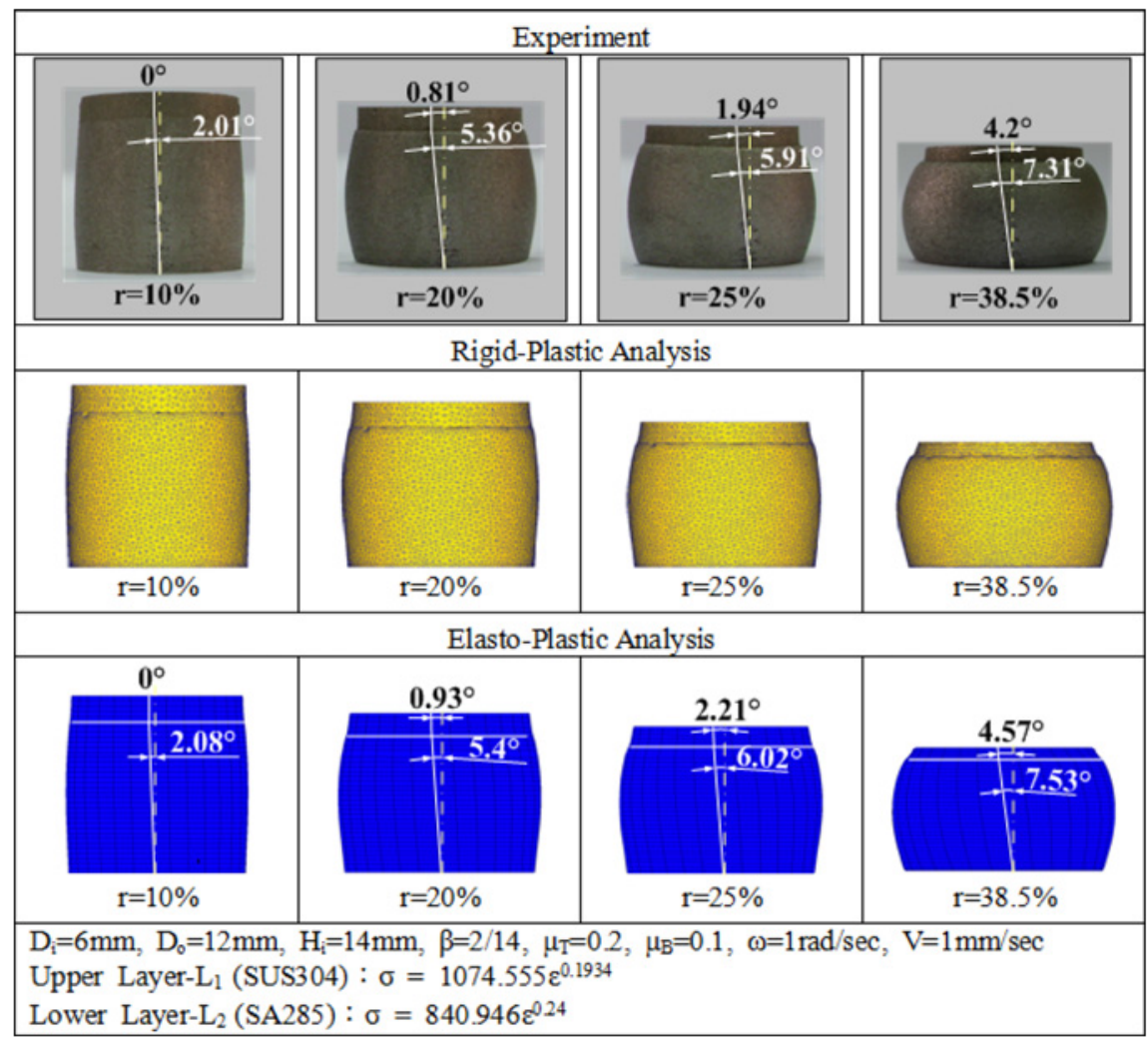

Fig. 10 Bulging profiles of outer surface and shear strain in the rotating forming with different reduction ratios

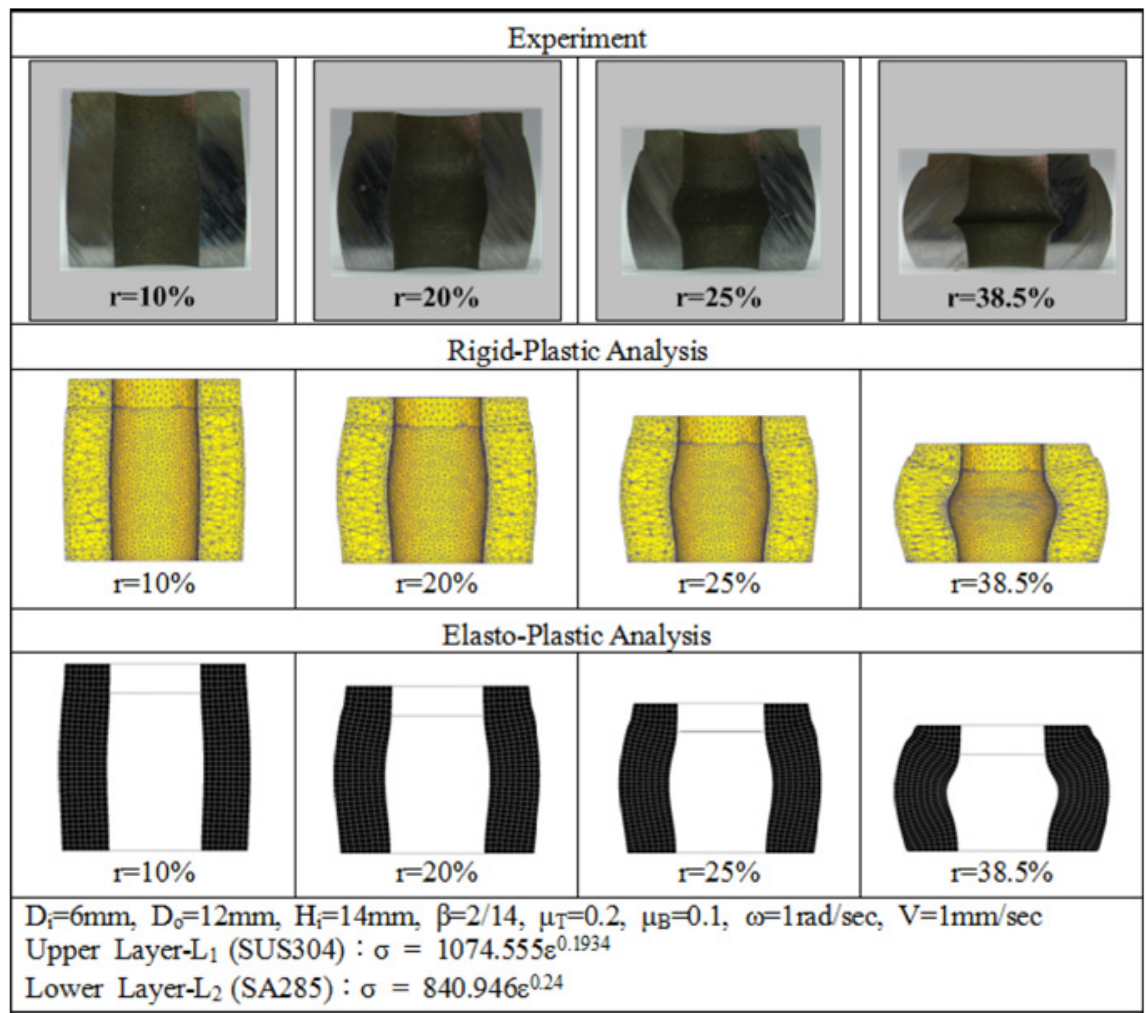

Fig. 11 Outer and inner deformation profiles in the rotating forming with different reduction ratios 
the reduction ratio increases, the shear strain is getting larger, as shown in experiment and elasto-plastic analysis simulation.

Due to the upper layer difficult to be deformed, the shear strain is not obvious; however that of the soft layer is. At $r=10 \%$, the shear strain is $0^{\circ}$ for hard layer, the lower layer is $2.01^{\circ}$ in the experiment; it is $0^{\circ}$ for hard layer and the lower layer (the soft layer) is $2.08^{\circ}$. The errors between both are $0 \%$ and $2.54 \%$ respectively. At $r=20 \%$, the shear strain is $0.81^{\circ}$ for hard layer; the lower layer is $5.36^{\circ}$ in the experiment; it is $0.93^{\circ}$ for hard layer and the lower layer is $5.4^{\circ}$. The errors between both are $5.36 \%$ and $0.75 \%$ respectively. At $\mathrm{r}=25 \%$, the shear strain is $1.94^{\circ}$ for hard layer; the lower layer is $5.91^{\circ}$ in the experiment; it is $2.21^{\circ}$ for hard layer and the lower layer is $6.02^{\circ}$. The errors between both are $5.91 \%$ and $1.86 \%$ respectively. At $r=38.5 \%$, the shear strain is $4.2^{\circ}$ for hard layer; the lower layer is $7.31^{\circ}$ in the experiment; it is $4.5^{\circ}$ for hard layer and the lower layer is $7.53^{\circ}$. The errors between both are $8.81 \%$ and $3.01 \%$ respectively.

Fig. 11 is the outer and inner deformation profiles in the rotating forming with different reduction ratios. From this table, because the

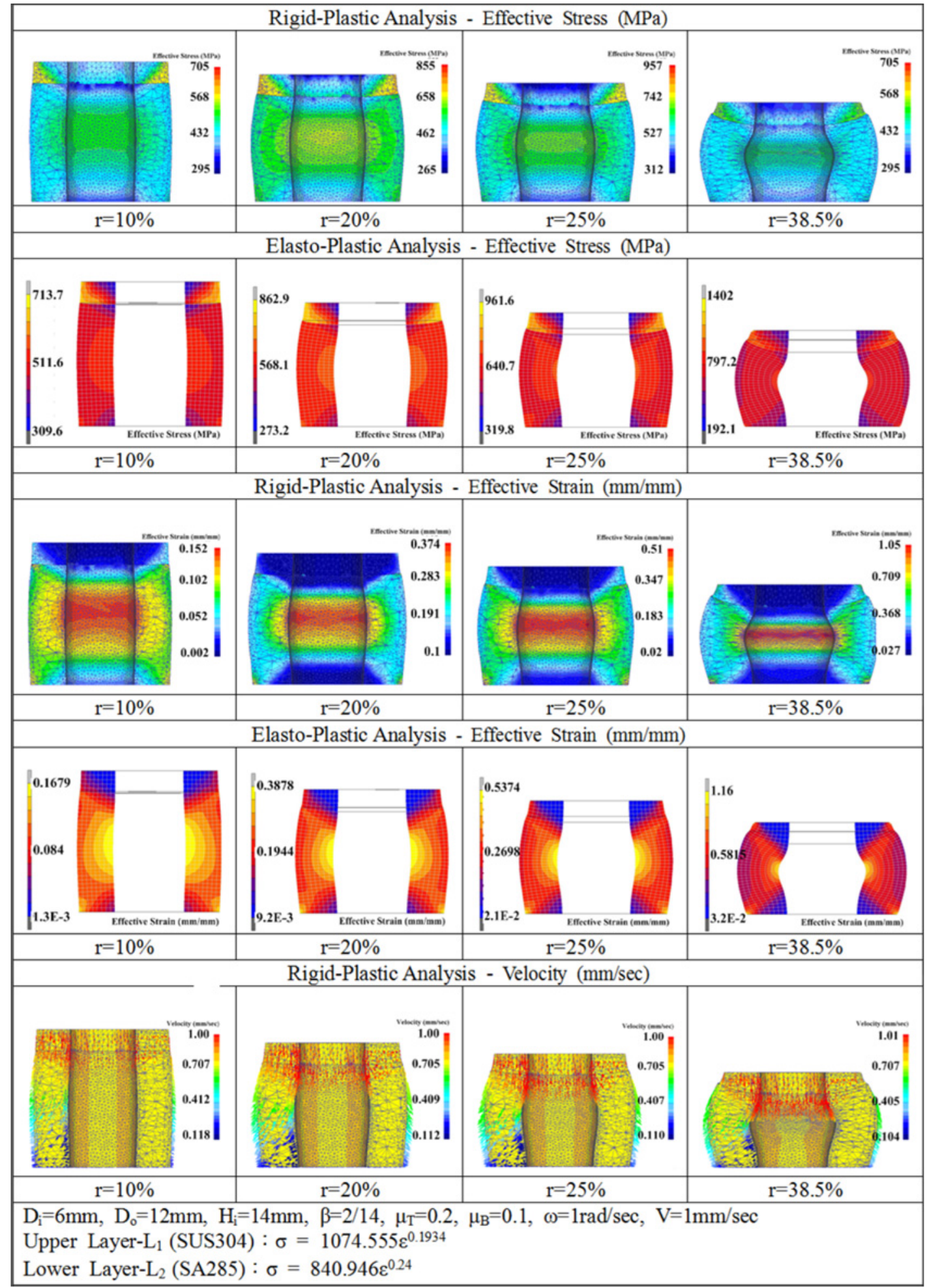

Fig. 12 Effective stress, effective strain in the rotating forming with different reduction ratios 
upper layer (SUS 304) is harder than the lower layer (SA258), the hard layer is inset to the soft layer. The soft layer is easy to be deformed, however the hard layer is difficult to deform so that it insets to the soft layer, as can be seen in experiments and both simulations. Moreover the lower layer has low frictional coefficient and the rotating die, thus the outer and inner surfaces are deformed away from the center of the clad ring and more uniform than that without rotation. And the compression is lower that without rotation. Both simulations are in good agreement with the experiments.

Effective stress and effective strain in the non-rotating forming with different reduction ratios are shown in Fig. 12. At $r=10 \%$, the maximum effective stress obtained from Deform 3D is $705 \mathrm{MPa}$, that obtained from elasto-plastic analysis is $713.7 \mathrm{MPa}$; the error between both simulations is around $1.12 \%$. At $r=20 \%$, the maximum effective stress obtained from rigid-plastic analysis is $855 \mathrm{MPa}$, that obtained from elasto-plastic analysis is $762.9 \mathrm{MPa}$; the error between both simulations is around $0.92 \%$. At $r=25 \%$, the maximum effective stress obtained from rigid-plastic analysis is $957 \mathrm{MPa}$, that obtained from elasto-plastic analysis is $961.6 \mathrm{MPa}$; the error between

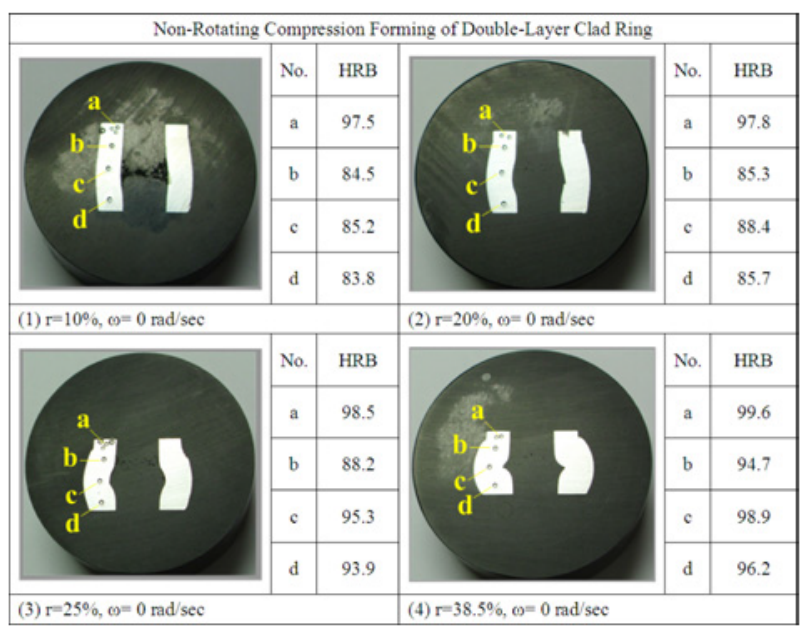

Fig. 13 The hardness under various reductions in non-rotating compression forming

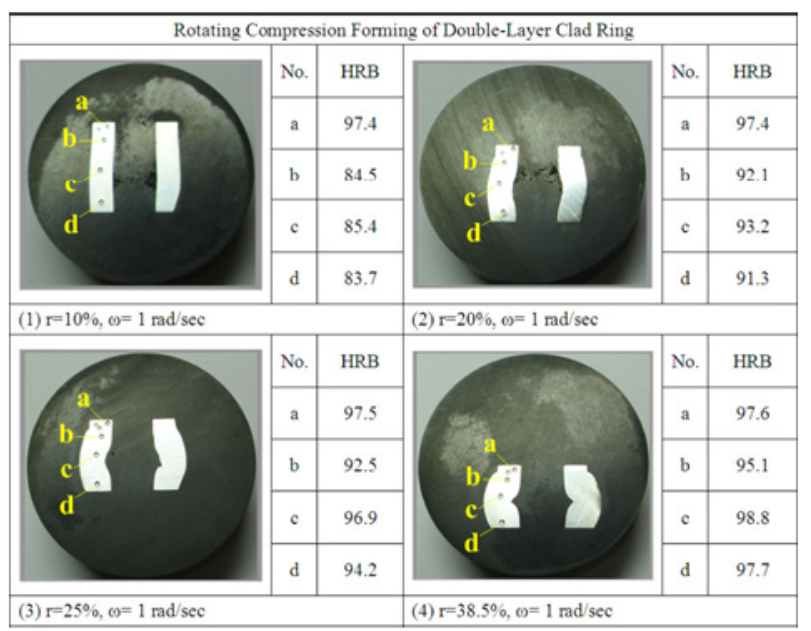

Fig. 14 The hardness under various reductions in rotating compression forming both simulations is around $0.44 \%$. At $r=38.5 \%$, the maximum effective stress obtained from rigid-plastic analysis is $1390 \mathrm{MPa}$, that obtained from elasto-plastic analysis is $1402 \mathrm{MPa}$; the error between both simulations is around $0.86 \%$. At $r=10 \%$, the maximum effective strain obtained from rigid-plastic analysis is $0.152 \mathrm{~mm} / \mathrm{mm}$, that obtained from elasto-plastic analysis is $0.17 \mathrm{~mm} / \mathrm{mm}$; the error between both simulations is very small. At $r=20 \%$, the maximum effective strain obtained from Deform $3 \mathrm{D}$ is $0.374 \mathrm{~mm} / \mathrm{mm}$, that obtained from elasto-plastic analysis is $0.39 \mathrm{~mm} / \mathrm{mm}$. At $\mathrm{r}=25 \%$, the maximum effective strain obtained from Deform $3 \mathrm{D}$ is $0.51 \mathrm{~mm} / \mathrm{mm}$, that obtained from elasto-plastic analysis is $0.54 \mathrm{~mm} / \mathrm{mm}$; the error between both simulations is around $0.85 \%$. At $r=38.5 \%$, the maximum effective strain obtained from rigid-plastic analysis is $1.05 \mathrm{~mm} / \mathrm{mm}$, that obtained from elasto-plastic analysis is $1.16 \mathrm{~mm} /$ $\mathrm{mm}$; the error between both simulations is around $9.48 \%$. The locations of maximum effective strain and stress are mainly on the inner surface deformed from the center of clad ring.

Finally, the hardness under various reductions in non-rotating compression forming can be explored in Fig. 13. The hardness of upper layer (SUS 304) is higher than the lower layer (the soft layer, SA285). However, the hardness of lower layer increases with the reduction, especially the point $\mathrm{c}(\mathrm{r}=38.5 \%$, HRB $=98.9)$.

The hardness under various reductions in rotating compression forming can be explored in Fig. 14. The hardness of upper layer (SUS 304) is still higher than the lower layer (the soft layer, SA285). However, the hardness of lower layer increases with the reduction, especially the point $\mathrm{c}(\mathrm{r}=38.5 \%$, HRB $=98.8)$. The average hardness for the lower layer in rotating compression is higher than that in nonrotating compression. That is because the twist effect will reduce the grain size to increase the hardness.

\section{Conclusions}

Through a series of simulations, the main results can be summarized as follows:

1. The Deform software using tetrahedron element and implicit FEM solver needs to take plenty of CPU time even adopting rigid-plastic analysis (one case 72 hours); however the Superform software using hexagonal element and FVM just takes a few CPU time even adopting elasto-plastic analysis (one case 10 minutes).

2. The shear strains can be measured form the Superform software and the experiment, and the maximum error is reasonable, around $9 \%$. However, the shear strain cannot be measured form Deform software due to the tetrahedron element.

3. The lower friction on the bottom die will make the inner and out surface of soft layer deform outward meanwhile.

4. Variations of rigid-plastic analysis, elasto-plastic analysis with experiments can be explored effectively.

5. The average hardness with rotation is higher than non-rotation.

The effective stress, the effective strain, the velocity field, the compressive force, the bulging, the shear strain can be explored by FEM simulations, and experimental verifications have been done in this study. 


\section{REFERENCES}

1. Douglas, J. and Altan, T., "Flow Stress Determination for Metals at Forging Rates and Temperatures," Journal of Engineering for Industry, Vol. 97, No. 1, pp. 66-76, 1975.

2. Sofuoglu, H. and Rasty, J., "On the Measurement of Friction Coefficient Utilizing the Ring Compression Test," Tribology International, Vol. 32, No. 6, pp. 327-335, 1999.

3. Kemin, X., Zhen, W., and Yan, L., "Fem Analysis of Cylinder Twist-compression Deformation Regularity," Joumal of Materials Processing Technology, Vol. 69, No. 1, pp. 148-151, 1997.

4. Kim, Y. H., Park, J. H., and Jin, Y. E., "An Analysis of Plastic Deformation Processes for Twist-assisted Upset Forging of Cylindrical Billets," Proc. of the Institution of Mechanical Engineers, Part B: Journal of Engineering Manufacture, Vol. 215, No. 6, pp. 883-886, 2001.

5. Kim, Y. H., Park, J. H., and Jin, Y. E., "An Upper Bound Analysis for Torsional Upset Forging of Cylindrical Billets," Proc. of the $6^{\text {th }}$ International Conference on Technology of Plasticity, Advanced Technology of Plasticity, Vol. 2, pp. 859-864, 1999.

6. Kim, Y. H., Jin, Y. E., Park, J. H., and Lee, Y., "An Analysis of the Torsional Forming Process using the Dual Stream Function," Proc. the $8^{\text {th }}$ International Conference on Metal Forming, pp. 741-745, 2000 .

7. Huang, M. and Tzou, G., "Study on Compression Forming of a Rotating Disk Considering Hybrid Friction," Journal of Materials Processing Technology, Vol. 125-126, No. pp. 421-426, 2002.

8. Huang, M. N., Tzou, G. Y., and Chen, C. J., "New Corresponding Relationships between Frictional Coefficient and Frictional Factor in Compression Forming of a Rotating Circular Disk," Key Engineering Materials, Vol. 274-276, pp. 409-414, 2004.

9. Tzou, G. Y., Huang, M. N., and Chen, C. J., "An Analytical Approach to Rotating Compression Forming of Circular Disk Considering Constant Shear Friction," Proc. of the Plasticity, pp. 445-447, 2002.

10. Tzou, G, Huang, M., Hwang, Y., and Hu, H., "Analysis and Experimental Verification on the Rotating Compression Forming of Cylinder," Journal-chinese Society Of Mechanical Engineers, Vol. 27, No. 6, pp. 687, 2006.

11. Tzou, G Y., Chien, W. T., Huang, M. N., and Tsai, D. L., "Slab Analysis of Rotating Compression Forming of Cylinder with Coulomb Friction," Proc. of the $5^{\text {th }}$ Conference on Precision Machinery and Manufacturing Technology., Vol. 125-126, pp. 421426, 2007.

12. Tzou, G. Y., Chien, W. T., Huang, M. N., and Tsai, D. L., "Analytical Model of Rotating Compression Forming of Cylinder with Hybrid Friction," Proc. of the $24^{\text {th }}$ National Conference on Mechanical Engineering the Chinese Society of Mechanical Engineers, pp. 3419-3424, 2007.
13. Chang, C. C., Tzou, G Y., Chien, W. T., and Tsai, D. L., "Investigation on the Rotating Compression Forming of Cylinder With Constant Shear Friction," Proc. of the $31^{\text {th }}$ National Conference on Theoretical and Applied Mechanics, pp. J20-01-J2005, 2007.

14. Hsu, H. H. and Tzou, G Y., "Two Analytical Models of Doublelayer Clad Sheet Compression Forming based on the Upper Bound and the Slab Methods," Joumal of Materials Processing Technology, Vol. 140, No. 1, pp. 604-609, 2003.

15. Alexandrov, S., Tzou, G. Y., and Hsia, S. Y., “A New Upper Bound Solution for a Hollow Cylinder Subjected to Compression and Twist," Proc. of the Institution of Mechanical Engineers, Part C: Joumal of Mechanical Engineering Science, Vol. 218, No. 4, pp. 369-375, 2004.

16. Chen, C. J., Tzou, G. Y., and Huang, M. N., "Study on the Twist Compression Forming of Cylinder based on the Upper Bound and Slab Methods," Journal of Materials Processing Technology, Vol. 174, No. 1, pp. 266-271, 2006.

17. Solhjoo, S., "A Note on "Barrel Compression Test": A Method For Evaluation of Friction," Computational Materials Science, Vol. 49, No. 2, Pp. 435-438, 2010.

18. Alexandrov, S., "An Analysis of the Axisymmetric Compression of Viscous Materials," Journal of Materials Processing Technology, Vol. 105, No. 3, pp. 278-283, 2000.

19. Wu, M. C. and Yeh, W. C., "Effect of Natural Boundary Conditions on the Upper-bound Analysis of Upset Forging of Ring and Disks," Materials \& Design, Vol. 28, No. 4, pp. 1245-1256, 2007.

20. Park, J., Kim, Y., and Bae, W., "An Upper-bound Analysis of Metal Forming Processes by Nodal Velocity Fields using a Shape Function," Journal of Materials Processing Technology, Vol. 72, No. 1, pp. 94-101, 1997. 